\title{
An Orthogonal and pH-Tunable Sensor-Selector for Muconic Acid Biosynthesis in Yeast
}

Snoek, Tim; Romero-Suarez, David; Zhang, Jie; Ambri, Francesca; Skjoedt, Mette L.; Sudarsan, Suresh; Jensen, Michael K.; Keasling, Jay D.

\section{Published in:}

A C S Synthetic Biology

Link to article, DOI:

10.1021/acssynbio.7b00439

Publication date:

2018

Document Version

Publisher's PDF, also known as Version of record

Link back to DTU Orbit

Citation (APA):

Snoek, T., Romero-Suarez, D., Zhang, J., Ambri, F., Skjoedt, M. L., Sudarsan, S., Jensen, M. K., \& Keasling, J. D. (2018). An Orthogonal and $\mathrm{pH}$-Tunable Sensor-Selector for Muconic Acid Biosynthesis in Yeast. A C S Synthetic Biology, 7(4), 995-1003. https://doi.org/10.1021/acssynbio.7b00439

\section{General rights}

Copyright and moral rights for the publications made accessible in the public portal are retained by the authors and/or other copyright owners and it is a condition of accessing publications that users recognise and abide by the legal requirements associated with these rights.

- Users may download and print one copy of any publication from the public portal for the purpose of private study or research.

- You may not further distribute the material or use it for any profit-making activity or commercial gain

- You may freely distribute the URL identifying the publication in the public portal 


\title{
An Orthogonal and pH-Tunable Sensor-Selector for Muconic Acid Biosynthesis in Yeast
}

\author{
Tim Snoek, ${ }^{\dagger}$ David Romero-Suarez, ${ }^{\dagger}$ Jie Zhang $^{\dagger}{ }^{\dagger}$ Francesca Ambri, ${ }^{\dagger}$ Mette L. Skjoedt, ${ }^{\dagger}$ Suresh Sudarsan, ${ }^{\dagger}$ \\ Michael K. Jensen, ${ }^{* \dagger \odot}$ and Jay D. Keasling ${ }^{\dagger,+, \&^{\prime}, \|}$ \\ ${ }^{\dagger}$ Novo Nordisk Foundation Center for Biosustainability, Technical University of Denmark, 2800 Kgs. Lyngby, Denmark \\ ${ }^{\ddagger}$ Joint BioEnergy Institute, Emeryville, California 94608, United States \\ ${ }^{\S}$ Biological Systems and Engineering Division, Lawrence Berkeley National Laboratory, Berkeley, California 94720, United States \\ "Department of Chemical and Biomolecular Engineering \& Department of Bioengineering, University of California, Berkeley, \\ California 94720, United States
}

\section{Supporting Information}

ABSTRACT: Microbes offer enormous potential for production of industrially relevant chemicals and therapeutics, yet the rapid identification of high-producing microbes from large genetic libraries is a major bottleneck in modern cell factory development. Here, we develop and apply a synthetic selection system in Saccharomyces cerevisiae that couples the concentration of muconic acid, a plastic precursor, to cell fitness by using the prokaryotic transcriptional regulator BenM driving an antibiotic resistance gene. We show that the sensor-selector does not affect production nor fitness, and find that

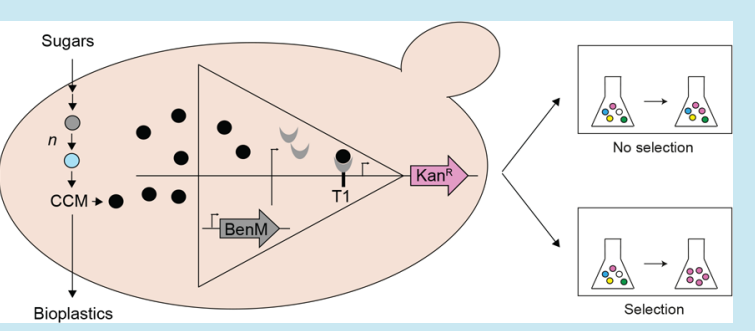
tuning $\mathrm{pH}$ of the cultivation medium limits the rise of nonproducing cheaters. We apply the sensor-selector to selectively enrich for best-producing variants out of a large library of muconic acid production strains, and identify an isolate that produces more than $2 \mathrm{~g} / \mathrm{L}$ muconic acid in a bioreactor. We expect that this sensor-selector can aid the development of other synthetic selection systems based on allosteric transcription factors.

KEYWORDS: transcriptional activator, biosensor, sustainability, evolution, metabolic engineering, yeast

\begin{abstract}
T $\mathrm{n}$ order to realize a biobased economy, metabolic engineering aims to develop microbes that can convert inexpensive, renewable feedstocks into valuable products. ${ }^{1}$ Initial genetically engineered strains, however, regularly need to be further optimized before their performance meets industrial demands on titers, rates and yields. Currently, decreases in DNA synthesis costs and the expansion of genome engineering tools allow for cost-effective building of large libraries of cell factory designs. $^{2,3}$ However, since the vast majority of chemicals targeted for overproduction in microbes are not coupled to easy selectable phenotypes, evaluation of individual strains often relies on low-throughput analytical methods, severely challenging the turn-around time of the design-build-test-learn cycle. ${ }^{4}$

In recent years, development within synthetic biology has enabled the design and application of allosterically regulated transcription factors as biosensors. ${ }^{5,6}$ Such one-component regulators are abundantly present in prokaryotes, ${ }^{7}$ and can convert intracellular concentrations of otherwise inconspicuous chemicals of interest into easily measurable outputs, such as fluorescence (sensor-reporters) and antibiotic resistance (sensor-selectors). ${ }^{4}$ Even in the yeast Saccharomyces cerevisiae, a well-established biotechnology workhorse, there is a large demand on improving current strains and generating yeasts that incorporate novel biosynthesis routes. ${ }^{8}$ To this end, a range of transcription factor-based biosensors that can aid the screening
\end{abstract}

of yeast cell factory variants have been described, ${ }^{9}$ including sensor-reporters for detection of xylose, ${ }^{10}$ malonyl-CoA, ${ }^{11}$ cis,cis-muconic acid (CCM) and naringenin. ${ }^{12}$ Although sensor-reporters have been used for selection for bestproducing cells by fluorescence-activated cell sorting (FACS) ${ }^{13,14}$ sensor-selectors can offer high-resolution coupling of chemical abundances with growth-selectable phenotypes in a simple and inexpensive manner. ${ }^{15}$ In prokaryotes, sensorselectors have been widely used to select best-performing microbial strains or to evolve microbes, ${ }^{16-18}$ but also in yeast a few examples have demonstrated coupling of production to growth through the expression of auxotrophic marker genes. ${ }^{19,20}$

Previously, we have shown that transcriptional activators belonging to the LysR-type transcriptional regulator (LTTR) family can successfully be transplanted into yeast and applied as small-molecule sensor-reporters. ${ }^{12}$ One of the sensor-reporters, BenM, enabled expression of GFP correlated to in vivo CCM production. CCM is a platform chemical that can be converted into adipic acid or terephtalic acid, which can be further polymerized into numerous plastics. ${ }^{21}$ Whereas the highest CCM titer to date has been ascribed to Escherichia coli, ${ }^{22}$ from a

Received: December 5, 2017

Published: April 3, 2018 


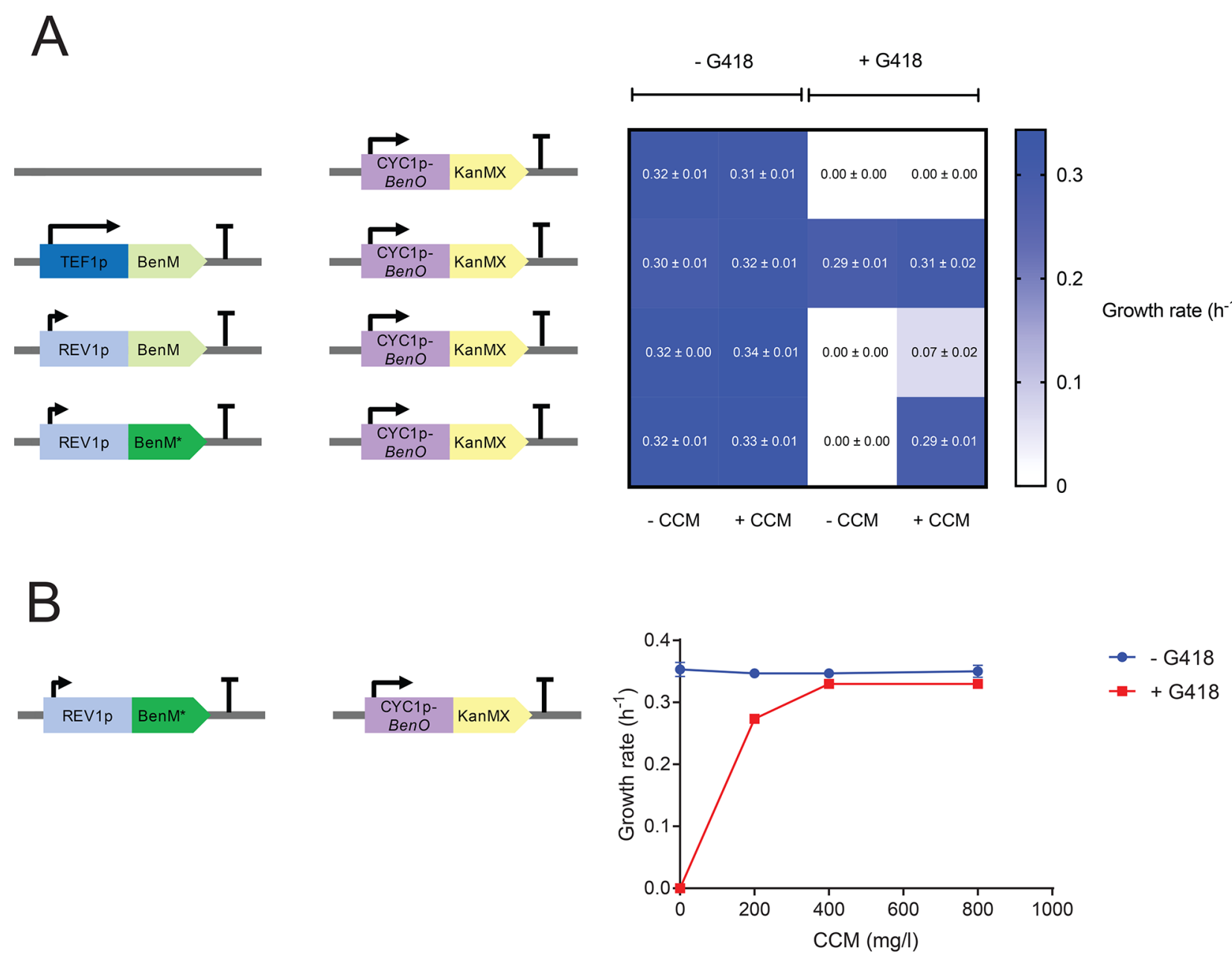

Figure 1. Characterization of biosensor designs for growth-coupled selection. (A) Four different strains harboring the selector construct (CYC 1p_BenO-kanMX) and no, high (TEF 1p), low (REV 1p) expression of wild-type BenM, or low expression (REV 1p) of a BenM triple mutant $\left(\mathrm{BenM}^{*}\right)$. Cells expressing either of these four designs were precultured in rich medium $\mathrm{pH} 4.5$ with or without $200 \mathrm{mg} / \mathrm{L} \mathrm{CCM}$, followed by subculturing into medium with the same composition with or without addition of $200 \mathrm{mg} / \mathrm{L} \mathrm{G} 418$. Growth was monitored during $24 \mathrm{~h}$. Growth rates are indicated in the heatmap as mean \pm standard deviation from three $(n=3)$ biological replicates. (B) The optimal sensor-selector design was tested in detail to determine the dose-response curve both in the presence and absence of G418. Growth rates are shown as mean \pm standard deviation from three $(n=3)$ biological replicates.

process point of view producing $\mathrm{CCM}$ in a low-pH tolerant organism such as $S$. cerevisiae is of great interest. Rational engineering as well as evolution have been applied to establish and improve CCM production in yeast. ${ }^{13,23-25}$ Notably, Leavitt and co-workers used a synthetic reporter promoter inducible by aromatic amino acids (AAAs) to drive the expression of an antibiotic resistance gene and evolve a strain with an increased pool of endogenous AAAs. ${ }^{13}$ Following two consecutive rounds of EMS mutagenesis and adaptive laboratory evolution for approximately $600 \mathrm{~h}$, the authors identified a strain producing $2.1 \mathrm{~g} / \mathrm{L}$ CCM.

In order to design and apply faster and more simple sensorselector systems based on small-molecule binding transcriptional activators, we re-engineered our previously identified CCM sensor-reporter design into a sensor-selector. First, we determined the optimal design for the sensor-selector, taking into account parameters such as biosensor expression level and dynamic range. Second, we showed that the sensor-selector does not affect the growth rate nor the performance of yeast engineered to produce CCM. Third, we demonstrated that tuning $\mathrm{pH}$ of the medium can be used to minimize the rise of fast-growing, yet low-producing, cheaters. Finally, we applied the sensor-selector to enrich for best-producing strains out of a large library of CCM production strains. From a large library of CCM pathway designs, we also showed biosensor-assisted selection of an isolate able to produce more than $2 \mathrm{~g} / \mathrm{L} \mathrm{CCM}$, on par with highest reported titers, and with higher productivity. To our knowledge, this is the first report on an orthogonal synthetic selection system in yeast driven by antibiotic resistance, which allows for rapid identification of best-producing cell factory variants from large strain libraries.

\section{RESULTS AND DISCUSSION}

Design and Characterization of a CCM SensorSelector. Previously we carried out a multiparametric analysis in order to develop a CCM biosensor based on the LysR-type transcriptional regulator BenM transplanted from Acinetobacter sp. ADP1 into S. cerevisiae. ${ }^{12}$ Here, we set out to develop a sensor-selector based in $S$. cerevisiae to couple chemical production to growth by replacing the reporter gene from our previous study with the $k a n M X$ gene, a widely used marker conferring resistance to the antibiotic G418. ${ }^{26}$ 


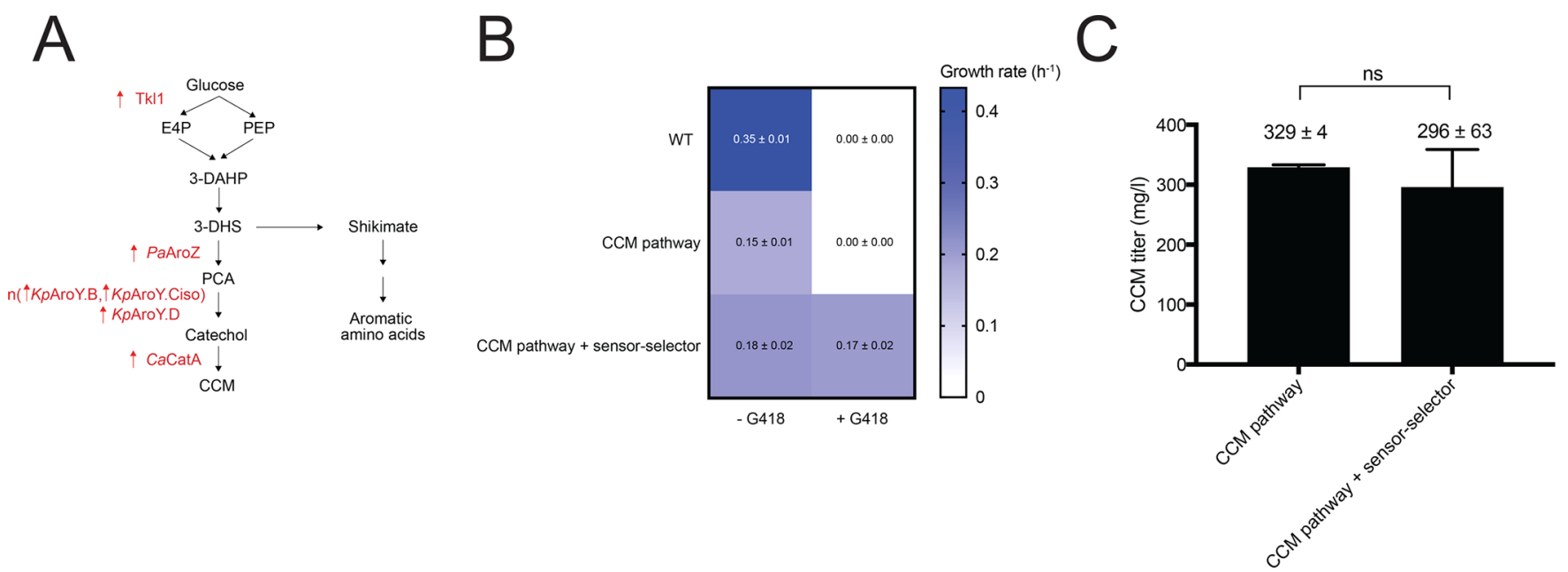

Figure 2. Biosensor-selector does not compromise cell factory performance. (A) A 3-step heterologous CCM pathway was built into yeast, comprising single-copy expression of $\mathrm{PaAroZ}, \mathrm{KpAroY} . \mathrm{D}$, and $\mathrm{CaCat} \mathrm{A}^{21}$ and multicopy integration of a cassette containing genes coding for KpAroY.B and KpAroY.Ciso. In addition, Tkl1 is overexpressed. (B) The sensor-selector was integrated into the CCM-producing strain (CCM pathway + sensor-selector) and its growth was compared to the baseline CCM-producing strain (CCM pathway) and wild-type CEN.PK. All three

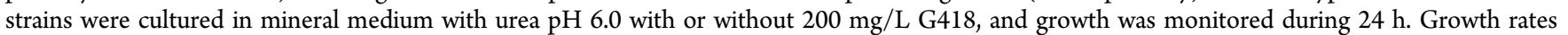
are indicated in the heatmap as mean \pm standard deviation from three $(n=3)$ biological replicates. (C) CCM titer was measured for the two CCM production strains after $72 \mathrm{~h}$ of cultivation in mineral medium with urea $\mathrm{pH}$ 6.0. Means and standard deviations from four $(n=4)$ biological replicates are shown. $\mathrm{ns}=$ not significant as evaluated by $t$-test $(p>0.05)$.

In order to identify an optimal sensor-selector design supporting CCM-dependent growth under selective conditions (i.e., G418), we first compared the growth rates of yeast strains harboring the selector ( $k a n M X$ driven by an engineered CYC1 promoter; CYC 1p_BenO_T1 ${ }^{12}$ ) combined with no BenM, BenM expressed from the strong TEF1 promoter, BenM expressed from the weak REV1 promoter, and a previously identified $^{12}$ BenM mutant (BenM*) expressed from the REV1 promoter. This mutant contains three amino acid substitutions (H110R, F211 V and Y286N) and displays an increased dynamic range as compared to wild-type BenM. ${ }^{12}$ First, each of the four strains expressing the $k a n M X$ cassette was precultured in medium with or without CCM, and then subcultured into medium with the same composition with or without G418. Here, we found that all four strains grew well in medium without selection, irrespective of the CCM concentration (Figure 1A). Contrastingly, without BenM, no growth was observed under selective conditions, whereas BenM expressed from the strong TEF1 promoter led to constitutive growth, even in the presence of G418 and absence of CCM. Finally, while BenM expressed from the weak REV1 promoter showed modest CCM-dependent growth under selective conditions, the strain expressing BenM* showed pronounced CCMdependent growth in the presence of G418. Therefore, REV $1 \mathrm{p}-B e n M^{*}$ combined with the selector was chosen as the optimal design. For this design, we found that when supplemented with $400 \mathrm{mg} / \mathrm{L} \mathrm{CCM}$, the growth rate was further increased under selective conditions, but no further increase was observed in the presence of $800 \mathrm{mg} / \mathrm{L}$ (Figure 1B). Finally, we found that introducing the sensor and/or the selector into yeast did not affect the basal growth rate both in medium with and without added CCM (Figure S1).

Taken together, these data demonstrate that the sensorselector design consisting of REV $1 \mathrm{p}-\mathrm{Ben}^{*}$ and the selector construct has the potential to couple accumulation of CCM to host growth without affecting the growth rate of yeast under nonselective conditions.
Sensor-Selector Validation in Production Strain. Next, we aimed to investigate if the sensor-selector would be functional in yeast engineered to produce CCM from a 3step heterologous biosynthetic pathway (Figure 2A). ${ }^{23,24} \mathrm{We}$ previously introduced this pathway into yeast and measured $\mathrm{CCM}$ production of individual variants differing in the number of an integrated cassette containing KpAroY.B and KpAroY.Ciso, genes encoding subunits of the rate-limiting enzyme AroY, which controls the conversion of protocatechuic acid (PCA) to catechol (see Figure 2A and Methods). ${ }^{12}$ We chromosomally integrated the sensor-selector in a strain (TISNO-11) that stably produced CCM based on a single integrated copy of the expression cassette for KpAroY.B-KpAroY.Ciso (Figure S9). In order to determine the effects of introducing the pathway and the sensor-selector into yeast, we measured growth and CCM production of this strain. To maintain selection for the production pathway (see Methods), these assays were carried out using mineral medium. Whereas the standard nitrogen source of this medium is ammonium sulfate, we replaced this with urea, since G418 is not effective in combination with ammonium sulfate. ${ }^{27}$ From this study we found no significant difference in growth rate between the original CCM-producing strain (CCM pathway) and the strain further engineered to express the sensor-selector (CCM pathway + sensor-selector) under nonselective conditions, though the CCM production strain grew significantly slower than wild-type CEN.PK ( $t$-test, $p<0.05)$, underscoring the growth burden of the production pathway (Figure 2B). As expected, the production strain without the sensor-selector was not able to grow in selective medium, whereas the production strain with the sensor-selector showed robust growth in the presence of G418 (Figure 2B). Moreover, there was no significant difference in CCM titer between the two strains (Figure 2C). Finally, we observed no changes in cell morphology as observed during $72 \mathrm{~h}$ cultivations of WT, CCM pathway, and CCM pathway + sensor-selector strains (Figure S2). 


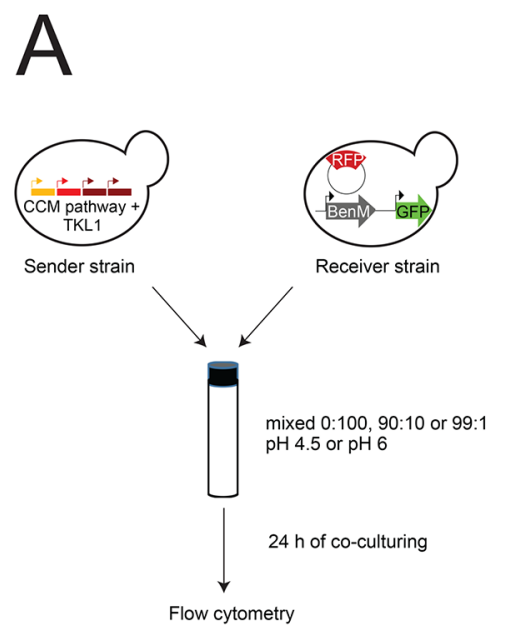

\section{B}

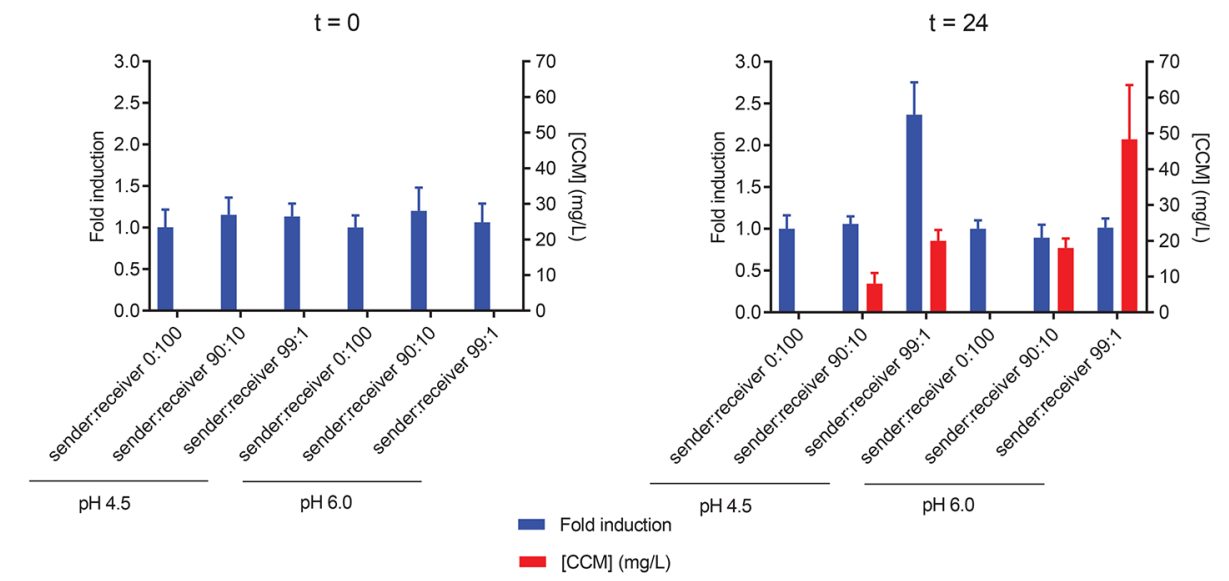

Figure 3. Safe-guarding selection from cheaters by $\mathrm{pH}$ control. (A) Outline of experimental setup. A CCM-producing strain (sender) was cocultured with a biosensor-reporter strain (receiver, no CCM production) in different inoculum ratios in mineral medium with ammonium sulfate at $\mathrm{pH} 4.5$ or $\mathrm{pH}$ 6.0. The receiver strain harbored a plasmid expressing RFP in order to identify biosensor cells by flow cytometry. (B) Biosensor activity and CCM production in cocultures at the onset $(t=0)$ and after $24 \mathrm{~h}(t=24)$ of culturing. Fold induction indicates the fold-change difference in mean GFP fluorescence intensity of cocultures relative to the sender:receiver 0:100 coculture with the same $\mathrm{pH}$ of $10000 \mathrm{RFP}^{+}$-cells per coculture. Fold induction and $[\mathrm{CCM}]$ are shown as mean \pm standard deviation from three biological replicates $(n=3)$ per coculture.

Taken together, these results show that the sensor-selector confers a growth-selectable phenotype when introduced to CCM-producing yeast without affecting CCM production, growth rate or cell morphology.

pH Tuning of the Sensor-Selector System. One of the major considerations for bulk screenings of large diverse populations of cell factory variants is the rise of false-positives; i.e., cells that do not produce the compound of interest but are still able to thrive under selective conditions. ${ }^{13,17}$ This is especially relevant for biosynthetic pathways where production confers a growth burden, as observed for CCM (Figure 2B). Due to the fact that protonated CCM can passively diffuse across the yeast cell membrane, ${ }^{12}$ we expected that one prominent way for cheaters to arise and be isolated from large genetic screens, would be for nonproducing fast-growing cells to take up CCM secreted by slow-growing producing cells, resulting in sensor-selector activation. We hypothesized that tuning the $\mathrm{pH}$ of the growth medium could control the rise of cheaters. CCM is a weak acid with a $\mathrm{p} K_{\mathrm{a}}$ of 3.57 , hence at $\mathrm{pH}$ levels close to this value, a higher fraction of the CCM molecules is uncharged and can diffuse into the cell, whereas at higher $\mathrm{pH}$ this fraction is smaller. ${ }^{28}$ Indeed, by external administration of CCM to the medium, we found that our previously described CCM sensor-reporter (REV $1 \mathrm{p}-B e n M^{*}$ in combination with the $y E G F P$ reporter gene) shows high induction at $\mathrm{pH} 4.5$, but no induction at $\mathrm{pH} 6.0$ (Figure S3).

In order to further test this hypothesis, cocultures of the nonproducing CCM sensor-reporter strain ("receiver strain") and the CCM production strain TISNO-11 ("sender strain") were performed. To track the relative abundance of the two strains, the receiver strain was also transformed with a plasmid expressing a gene encoding red fluorescent protein (RFP) from the TEF2 promoter (Figure 3A). Cocultures were performed in mineral medium with ammonium sulfate brought to $\mathrm{pH} 4.5$ or pH 6.0 in three different sender:receiver starting ratios: 0:100, 90:10 and 99:1. At the onset of the cocultures $(t=0)$ and after $24 \mathrm{~h}(t=24)$ the fraction of the population consisting of the receiver strain (RFP), and the sensor-controlled reporter gene activity (GFP) in those cells was determined using flow cytometry (Figure S4A). For the sender:receiver 0:100 culture, we found that the percentage of RFP-expressing cells to be around $80-90 \%$ at both $t=0$ and $t=24$ (Figure S4B), probably due to expression fluctuations caused by plasmidbased expression (Figure S4A). Most, importantly, as inferred from percentages of RFP-expressing cells, we found that population distributions for the three different ratios looked similar independent of the $\mathrm{pH}$ applied (Figure S4B). On the contrary, for cells cultivated at $\mathrm{pH} 4.5$, the CCM-inducible biosensor induced GFP expression 2.4-fold, whereas no induction was observed at pH 6.0 (Figure 3B). Interestingly, quantification of CCM production in cocultures pointed out that CCM levels were higher in $\mathrm{pH} 6.0$ (approximately 18-49 $\mathrm{mg} / \mathrm{L}$ ) than in $\mathrm{pH} 4.5$ (approximately $8-20 \mathrm{mg} / \mathrm{L}$ ) (Figure 3B). By measuring the final $\mathrm{pH}$ we found a severe $\mathrm{pH}$ drop for all cultures: cultures started at $\mathrm{pH} 4.5$ reached a final $\mathrm{pH}$ of 3.0 \pm 0.0 , whereas the $\mathrm{pH}$ of cultures started at $\mathrm{pH} 6.0$ dropped down to $\mathrm{pH} 4.9 \pm 0.1$ (Figure S4C).

These data show that $\mathrm{pH}$ of the growth medium can control the degree of passive diffusion of CCM into nonproducing cells, and that $\mathrm{pH}$ can provide a simple tuning parameter for bulk screening and selection of production strain libraries.

High-Throughput Screening of a CCM Production Strain Library. In order to determine whether the sensorselector is able to enrich for high CCM-producing variants when grown in batch, we created a library of CCM-producing strains using a semirandomized approach. As a starting strain, we used a strain overexpressing the TKL1 gene encoding transketolase 1 in addition to the CCM biosynthetic pathway consisting of genes coding for PaAroZ, KpAroY.D and CaCatA. ${ }^{12}$ This strain does not produce detectable amounts of CCM. ${ }^{12}$ We first integrated the sensor-selector into this strain, and following transformation of an expression cassette harboring KpAroY.B and KpAroY.Ciso for multicopy integration into Ty4 sites, $^{29}$ we obtained a library of approximately $10^{4}$ transformants (see Methods). Next, these transformants were precultured in bulk, followed by inoculation of three different flasks with selective medium (selection, i.e., $200 \mathrm{mg} / \mathrm{L} \mathrm{G418),}$ as well as three flasks containing nonselective medium 

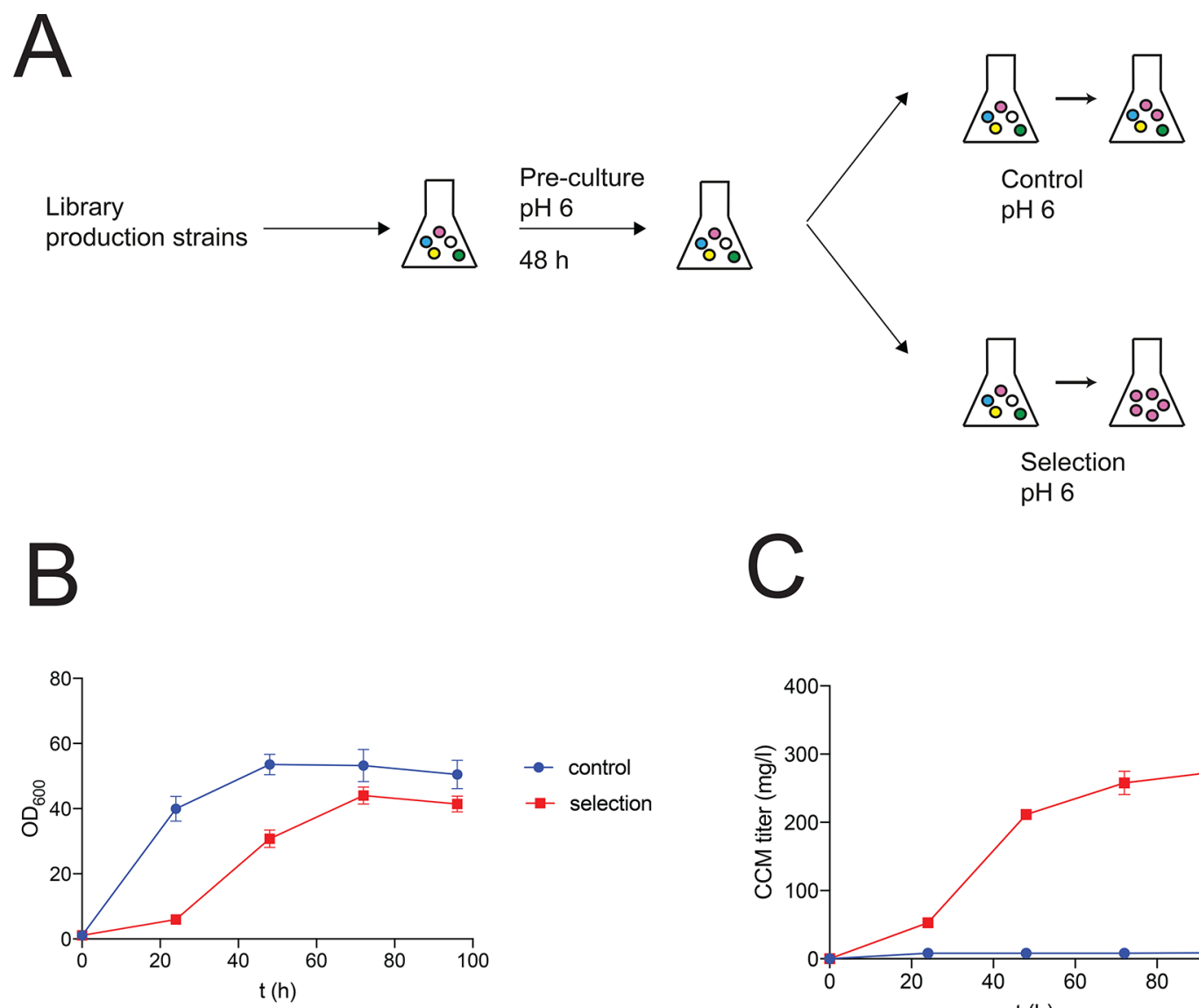

C

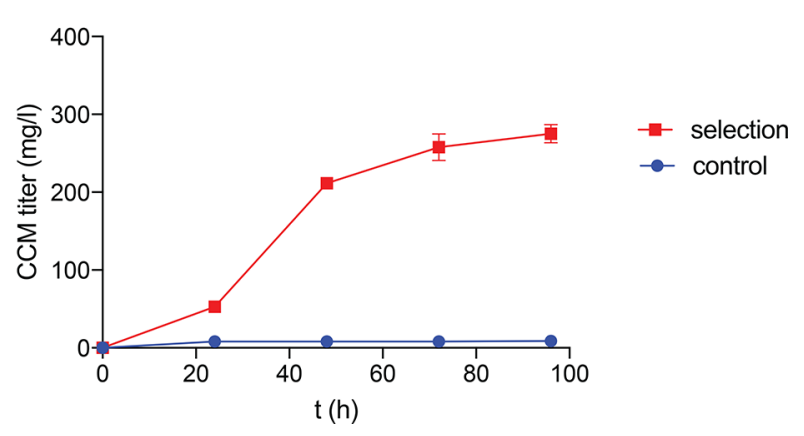

Figure 4. Pathway evolution using synthetic selection. (A) Experimental outline of multicopy AroY library screening using the sensor-selector. The library was based on transformation of a construct consisting of KpAroY.B and KpAroY.Ciso targeted to Ty4 sites into a strain containing the 3-step CCM production pathway and the sensor-selector. Library screening consisted of preculturing, followed by subculturing into mineral medium with urea pH 6 (control) and into mineral medium with urea pH $6+200 \mathrm{mg} / \mathrm{L}$ G418 (selection). (B) $\mathrm{OD}_{600}$ values of strain library cultivations with (selection) or without (control) G418 supplemented to the medium. (C) CCM titers from library cultivations. For both (B) and (C), means and standard deviations from three $(n=3)$ biological replicates per cultivation condition are shown.

(control) (Figure 4A). Whereas the control cultures grew to saturation within approximately $30 \mathrm{~h}$, the cultures growing under selective conditions needed more than $48 \mathrm{~h}$ to reach saturation (Figure 4B). We found that the $\mathrm{pH}$ of the cultures was maintained around 6 (see Figure S5 and Methods). Most importantly, whereas no CCM could be detected in the control cultures for any time point, a steady increase in CCM titer in the selective cultures, up to $275 \pm 12 \mathrm{mg} / \mathrm{L}$ after $96 \mathrm{~h}$, was observed (Figure 4C), proving the power of the sensor-selector to robustly, and in high-throughput, enrich for CCM-producing variants.

We hypothesized that before subculturing the cells into selective medium, a proportion of the population would already consist of nonproducing, fast-growing cells that have low copy numbers of KpAroY.B and KpAroY.Ciso. In order to verify this hypothesis, we characterized the starting population by measuring the growth with and without G418 of 89 single colonies isolated at the end of the $48 \mathrm{~h}$ preculture. From this, we observed a wide variation in growth rates in medium without G418, yet only two isolates were able to grow in the presence of G418 (Figure S6A). In order to verify whether the "G418+" phenotype correlated with CCM production, we focused on the isolates from the preculture, and measured CCM production for the two G418-positive clones, as well as for five isolates that were not able to grow in the presence of
G418 spanning different growth rates (Figure S6A). Only the two G418-positive clones (isolates 6 and 7) showed CCM production, whereas the remaining isolates (isolates 1-5) did not produce detectable amounts of CCM (Table 1). These data

Table 1. Overview of Growth Rates (See Figure S6A), Phenotype in Selective Medium (Mineral Medium with Urea pH $6.0+200 \mathrm{mg} / \mathrm{L} \mathrm{G418)}$ and CCM Production in Seven Library Isolates

\begin{tabular}{cccc} 
isolate & phenotype & growth rate mineral medium $\left(\mathrm{h}^{-1}\right)$ & $\mathrm{CCM}$ titer $(\mathrm{mg} / \mathrm{L})$ \\
1 & G418- & 0.34 & $0.3 \pm 0.1$ \\
2 & G418- & 0.29 & $0.3 \pm 0$ \\
3 & G418- & 0.33 & $0.3 \pm 0$ \\
4 & G418- & 0.19 & $0 \pm 0$ \\
5 & G418- & 0.21 & $0 \pm 0$ \\
6 & G418+ & 0.11 & $379 \pm 3$ \\
7 & G418+ & 0.14 & $470 \pm 41$ \\
\hline
\end{tabular}

show that right before applying selection, the library indeed consisted of a high proportion of nonproducing cells. Contrastingly, scoring G418 phenotypes postselection, we found all single colonies (90) to be G418-resistant (Figure $\mathrm{S} 6 \mathrm{~B})$. Interestingly, similar to the distribution of growth rates from colonies sampled preselection (Figure S6A), more than 2- 
A

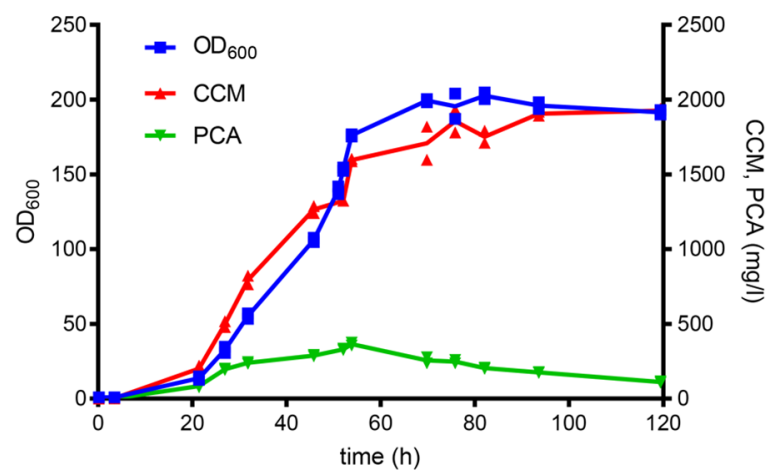

B

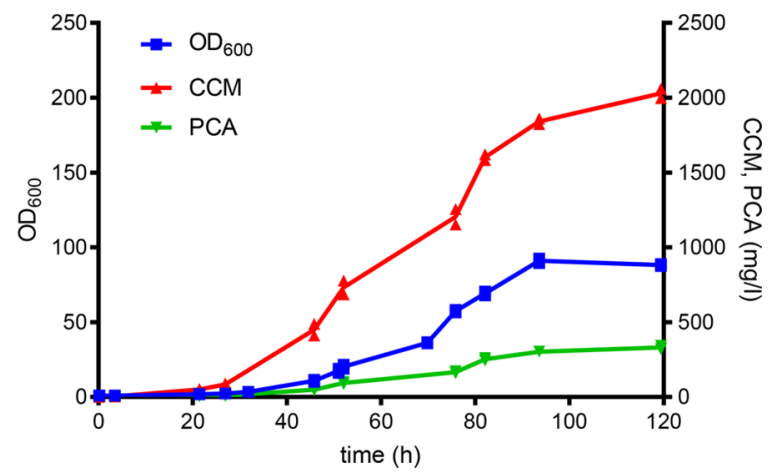

Figure 5. Bioreactor fermentations of selected CCM-producing strains Biomass units $\left(\mathrm{OD}_{600}\right)$, and titers of $\mathrm{CCM}$ and PCA from a repeated batch fermentation of isolate 6 (A) and isolate 7 (B) during a $120 \mathrm{~h}$ cultivation in mineral medium with urea pH 6.0. For both (A) and (B), the mean values and individual data points from two $(n=2)$ biological replicates are shown. See Figure S7 and Methods for details.

fold changes in growth rates were observed between slowest and fastest growing colonies sampled postselection (Figure S6B).

We next scaled-up CCM production of the two G418positive clones (i.e., isolates 6 and 7) in bioreactors in duplicates. In order to reach high titers, additional concentrated medium was spiked as soon as the $\mathrm{CO}_{2}$ production dropped, indicative of glucose depletion (see Figure S7). Both strains reached high titers, with isolate 6 reaching on average 1927 $\mathrm{mg} / \mathrm{L} \mathrm{CCM}$ (Figure 5A), and isolate 7 producing on average $2008 \mathrm{mg} / \mathrm{L} \mathrm{CCM}$ (Figure 5B). Isolate 6 displayed an average yield of $11.4 \mathrm{mg} \mathrm{CCM} / \mathrm{g}$ glucose with an average productivity of $23.8 \mathrm{mg} \mathrm{CCM} /\left(\mathrm{g}\right.$ glucose $\left.\mathrm{h}^{-1}\right)$, and isolate 7 showed an average yield of $13.4 \mathrm{mg} \mathrm{CCM} / \mathrm{g}$ glucose with an average productivity of $19.9 \mathrm{mg} \mathrm{CCM} /\left(\mathrm{g}\right.$ glucose $\mathrm{h}^{-1}$ ) (see Figure S8 and Methods).

In order to further characterize the two strains, we sequenced the full genomes of these production strains to determine the copy number of the KpAroY.B-KpAroY.Ciso expression cassette in each isolate (see Methods). Here we found that copy number correlated with CCM production; with the topproducing isolate 7 bearing seven copies, and isolate 6 displaying six copies (Figure S9).

In summary, in this study, we designed, characterized and applied a fast and simple sensor-selector system in S. cerevisiae that directly couples the concentration of a chemical produced by a single cell to its fitness. Since BenM is part of the LTTR superfamily of small molecule-inducible prokaryotic transcriptional regulators, ${ }^{30}$ we envision that the sensor-selector system developed in this study could serve as a blueprint to develop high-throughput synthetic selection systems for a multitude of compounds regulated by LTTR-based transcription factors. Ultimately, this will significantly increase the turn-around time of the design-build-test-learn cycle for engineering future microbial cell factories.

\section{METHODS}

Strains, Chemicals and Media. Yeast strains were grown in rich (YPD), Synthetic Complete (SC) or mineral medium with urea (MMU) or ammonium sulfate (MMAS) as a nitrogen source. MMAS was prepared as described previously, ${ }^{31}$ for MMU $2.3 \mathrm{~g} / \mathrm{L}$ urea (Sigma, U1250) was used as a nitrogen source in order for G418 to be effective. Also, the final $\mathrm{pH}$ was brought to 4.5 or 6.0 . To test the response of nonproducing cells to CCM (see further), cis,cis-muconic acid (Sigma, 15992) was freshly dissolved in rich or MMAS medium, after which the $\mathrm{pH}$ of the medium was brought to 4.5 or 6.0 and filtersterilized. CCM production strains were grown in mineral medium in order to maintain selection for the destabilized uracil marker. Saccharomyces cerevisiae CEN.PK113-5A (MATa, trp1 his3 11 leu2-3/112 MAL2-8 ${ }^{c}$ SUC2) and CEN.PK113-7D (wild type, MATa MAL2-8c SUC2) strains were obtained from Peter Kötter (Johann Wolfgang GoetheUniversity Frankfurt, Germany). CCM production strain TISNO-11 was obtained from an EasyCloneMulti integration of a cassette harboring KpAroY.B and KpAroY.Ciso as carried out previously, ${ }^{12}$ and was in this study found to harbor one copy of this cassette. Escherichia coli strain DH $5 \alpha$ was used as a host for cloning and plasmid propagation, and was grown at 37 ${ }^{\circ} \mathrm{C}$ in Luria-Bertani medium supplemented with $100 \mu \mathrm{g} / \mathrm{mL}$ ampicillin. Phusion High-Fidelity DNA Polymerase or Phusion U Hot Start DNA Polymerase was used for PCR amplification according to manufacturer's instructions.

Plasmids and Strain Construction. An overview of plasmids used and constructed in this study is supplied in Supplementary Table S1. The lithium acetate method was used to transform yeast cells, ${ }^{32}$ followed by selection of transformants on synthetic drop-out medium (Sigma-Aldrich). For selection of strains transiently expressing kanMX and natMX markers, $200 \mu \mathrm{g} / \mathrm{mL} \mathrm{G418} \mathrm{sulfate} \mathrm{(Sigma,} \mathrm{G8168)} \mathrm{and} 100 \mu \mathrm{g} /$ $\mathrm{mL}$ nourseothricin dihydrogen sulfate (WERNER BioAgents, product no. 5.0), respectively, were added to the medium. Genomic integrations were achieved using EasyClone plasmids ${ }^{31}$ or marker-free EasyClone plasmids in combination with plasmids containing dominant markers on Cas9 and gRNA plasmids. ${ }^{33,34}$ Transformants were genotyped using oligonucleotides described in Supplementary Table S2. The resulting strains are listed in Supplementary Table S3.

The sensor and selector constructs (NotI digested pTS-5 and pTS-7) were integrated into EasyClone sites X-3 and XII-4, respectively, into strain ST2377 and TISNO-11 using pCfB2312 for Cas9 and pTS-9 for gRNA expression, which were subsequently cured off, generating strains DRS16 and TISNO-33, respectively. DRS16 formed the basal strain for the CCM production strain library. ST2377 was described previously $^{12}$ and contains the dehydroshikimate DHS dehydratase from Podospora anserina ( $\mathrm{PaAroZ}$ ), the $\mathrm{D}$ subunit of the PCA decarboxylase from Klebsiella pneumoniae (KpAroY.D), 
and the catechol 1, 2 dioxygenase CDO from Candida albicans ( $\mathrm{CaCatA})$. As carried out previously, ${ }^{12}$ we inserted multiple copies of a cassette containing the genes KpAroY.B and KpAroY.Ciso, coding for respectively the $\mathrm{B}$ subunit and a homologue of the $\mathrm{C}$ subunit of the PCA decarboxylase from K. pneumoniae, $^{23}$ using the EasyCloneMulti system ${ }^{25}$ into DRS16. The library was obtained by adding all the cells posttransformation to a final volume of $25 \mathrm{~mL}$ SC-Ura, growing overnight, and storing cells in aliquots at $-80^{\circ} \mathrm{C}$. Immediately after transformation, a defined number of cells was plated onto SC-Ura in order to determine the number of transformants as a proxy for library size.

Library Enrichment. Ten $\mathrm{OD}_{600}$ units of the library (>6600 coverage) were added to a total volume of $25 \mathrm{~mL}$ MMU pH 6 (starting $\mathrm{OD}_{600}=0.4$ ) in $250 \mathrm{~mL}$-Erlenmeyer flasks and grown for $48 \mathrm{~h}$ at $250 \mathrm{rpm}, 30^{\circ} \mathrm{C}$ (preculture). After $48 \mathrm{~h}$ the preculture had reached $\mathrm{OD}_{600}=7.5$. At this stage a small portion of the preculture was plated for single colonies, of which 89 random clones were picked for growth rate determination. Biomass was harvested, centrifuged (5 min, $3000 \mathrm{~g}$ ) and supernatant removed, and used to inoculate three selective cultures ( $25 \mathrm{~mL}$ MMU pH $6+200 \mathrm{mg} / \mathrm{L} \mathrm{G418)}$ as well as three control cultures $(25 \mathrm{~mL}$ MMU pH 6$)$ to an initial $\mathrm{OD}_{600}=1.0$ and incubated at $30{ }^{\circ} \mathrm{C}, 250 \mathrm{rpm}$. The $\mathrm{OD}_{600}$ of the six cultures was determined on a daily basis for up to $96 \mathrm{~h}$ and every day $1 \mathrm{~mL}$ of broth was centrifuged and the supernatant saved for CCM quantification by HPLC and $\mathrm{pH}$ measurement. Biomass from the selective cultures was plated for single colonies, after which 90 random clones were picked for growth rate determination.

Growth Rate Determination. In different experiments the growth rate of yeast strains was determined. In order to assess the response curve to externally applied CCM of different sensor-selector designs, strains were grown overnight in $150 \mu \mathrm{L}$ YPD per well (preculture). The next day, precultures were subcultured 1:150 into either control medium (YPD pH 4.5) or YPD supplemented with CCM (40, 80, 120, 160, or $200 \mathrm{mg} / \mathrm{L})$ at $\mathrm{pH} 4.5$, followed by overnight growth. The next day, saturated cultures were diluted 1:150 into fresh medium with the same composition, with or without addition of $200 \mathrm{mg} / \mathrm{L}$ G418. CCM production strains were precultured in SC-Ura medium overnight, and subcultured 1:150 into MMU pH 6.0 with or without addition of $200 \mathrm{mg} / \mathrm{L}$ G418. Plates were sealed with Breathe-Easy sealing membrane (Sigma Z380059) and incubated at $30{ }^{\circ} \mathrm{C}$ in a platereader (BioTek ELx 808) with continuous shaking and $\mathrm{OD}_{630}$ measurements every $20 \mathrm{~min}$ for 24 or $72 \mathrm{~h}$. Growth rates were calculated using GATHODE software. $^{35}$ For each strain and condition at least three biological replicates were measured.

CCM Production Assays. For deep-well fermentations, strains were grown overnight in SC-Ura in a microtiter plate. The next day, the $\mathrm{OD}_{600}$ of the precultures were measured, and strains were subcultured to starting $\mathrm{OD}_{600}=1.0$ (approximately $10^{7}$ cells $/ \mathrm{mL}$ ) in $500 \mu \mathrm{L}$ MMU pH 6.0 in deep-well 96-well plates. After $72 \mathrm{~h}$ of incubation at $30{ }^{\circ} \mathrm{C}, 300 \mathrm{rpm}$, the final $\mathrm{OD}_{600}$ was measured, cells were centrifuged ( $5 \mathrm{~min}, 3000 \mathrm{~g}$ ), and the supernatant was used for HPLC quantification of CCM as described previously. ${ }^{12}$ Yeast cell morphology was evaluated with a Leica DM4000 B microscope equipped with a DFC 300 FX R2 camera (Leica Microsystems, Wetzlar, Germany).

For bioreactor cultivations, a procedure similar as previously described was followed. ${ }^{13}$ Two isolates, TISNO-219 (isolate 6) and TISNO-221 (isolate 7), were precultured in $50 \mathrm{~mL} \mathrm{SC-Ura}$ in duplicates in $250 \mathrm{~mL}$-Erlenmeyer flasks overnight. The next day, the $\mathrm{OD}_{600}$ of each preculture was measured, and a portion of biomass was harvested in order to inoculate 1-L bioreactors (Sartorius, Gottingen, Germany) to starting $\mathrm{OD}_{600}$ of 1.0. The starting medium of each bioreactor was $500 \mathrm{~mL}$ MMU pH 6.0 containing $4 \%(\mathrm{w} / \mathrm{v})$ glucose. During the cultivation, the stirring speed was maintained at $500 \mathrm{rpm}$ and the dissolved oxygen level was kept above $20 \%$ by cascaded mixing of pure oxygen to the air stream (air input flow rate of 0.5 standard liter per minute). The $\mathrm{pH}$ was controlled at 6.0 by addition of $7 \mathrm{M}$ $\mathrm{NaOH}$, and the temperature was maintained at $30{ }^{\circ} \mathrm{C}$. At regular intervals, samples were withdrawn for $\mathrm{OD}_{600}$ measurement, afterward centrifuged ( $5 \mathrm{~min}, 3000 \mathrm{~g}, 4{ }^{\circ} \mathrm{C}$ ) and the supernatant kept for HPLC analysis to determine CCM, PCA and glucose levels. During the fermentation, the off-gas $\mathrm{CO}_{2}$ production was monitored continuously (Thermo Scientific Prima BT MS). Sterile fresh medium (containing 50 or $100 \mathrm{~mL}$ of $10 \times \mathrm{MMU}$ medium) was pulsed after observing a significant drop in $\mathrm{CO}_{2}$ levels. In total $350 \mathrm{~mL} 10 \times \mathrm{MMU}$ was added.Fermentations were performed for a total period of 5 days. The yield (mg produced CCM/g consumed glucose) for each time point was calculated by dividing the CCM concentration $(\mathrm{mg} / \mathrm{L})$ by consumed glucose $(\mathrm{g} / \mathrm{L})$. The volumetric productivity ( $\mathrm{mg}$ produced $\mathrm{CCM} /(\mathrm{h} \mathrm{L})$ ) for each time point was calculated by dividing the CCM concentration $(\mathrm{mg} / \mathrm{L})$ by time $(\mathrm{h})$. Subsequently the yield and productivity for each strain replicate was calculated by taking the average of all time points excluding the first batch, i.e., from 31 to $120 \mathrm{~h}$ for isolate 6 , and from 69 to $120 \mathrm{~h}$ for isolate 7 (see Figure S7).

Flow Cytometry Analysis of CCM Administration Assays and Cocultures. To test the effect of external CCM on biosensor-reporter activation, our previously described strains MeLS0138 (reporter-only) and MeLS0284 (biosensorreporter) were subjected to growth in MMAS $\mathrm{pH} 4.5$ or $\mathrm{pH}$ 6.0, \pm supplementation of $200 \mathrm{mg} / \mathrm{L}$ CCM. After $24 \mathrm{~h}$ of growth the cells were diluted in PBS and analyzed by flow cytometry (MACSQuant Analyzer 10) with a blue laser (488 $\mathrm{nm}$ ) to detect yEGFP, FCS files were incorporated into FlowJo, and per replicate the mean GFP intensity of 10000 single-cell events was determined.

To determine the degree of biosensor-reporter activation in nonproducing cells, cocultures of sender strain TISNO-11 and receiver strain TISNO-31 were set up. Three single colonies of each strain were grown overnight in $3 \mathrm{~mL}$ SC-Ura-His-Leu-Trp. The next day the $\mathrm{OD}_{600}$ was measured, and cocultures were started in MMAS pH 4.5 or $\mathrm{pH} 6.0$ with a starting $\mathrm{OD}_{600}=0.2$. For each medium, sender and receiver strain were mixed in 0:100, 90:10 and 99:1 ratios in triplicates. After approximately $24 \mathrm{~h}$ cultures were diluted in PBS and analyzed on a BD Biosciences Aria (Becton Dickinson) with a blue laser (488 $\mathrm{nm})$ to detect yeGFP and a yellow green laser $(561 \mathrm{~nm})$ to detect mKate2. FCS files were incorporated into FlowJo, and per replicate the mean GFP intensity of $10000 \mathrm{RFP}+$ cells was determined after gating for single-cell events (see Figure S4A).

Genomic DNA Extraction. The genomic DNA (gDNA) of TISNO-11, TISNO-219 (isolate 6) and TISNO-221 (isolate 7) was extracted using the Yeast DNA Extraction Kit (Thermo Fisher) and final gDNA was eluted in $10 \mathrm{mM}$ Tris- $\mathrm{HCl}, \mathrm{pH} 8.0$. The final concentration of each sample was measured by the Qubit 2.0 Fluorometer and Qubit dsDNA HS assay Kit (Thermo Fisher), and afterward adjusted to a final concentration of $2 \mathrm{ng} / \mu \mathrm{L}$ in $50 \mu \mathrm{L}$ for genomic library preparation. 
The genomic libraries were generated using the KAPA HyperPlus Kit (Roche).

Whole Genome Sequencing and Copy Number Analysis. Illumina reads were aligned to the reference genome of S. cerevisiae CEN.PK113-7D (www.ncbi.nlm.nih.gov/ assembly?LinkName=bioproject_assembly_all\&from_uid= 52955) and to the integrative cassette sequences using bwa (PMID 19451168). Copy numbers for integrated genes (KpAroY.B and KpAroY.Ciso) were determined by comparing the GC-normalized read coverage of the integrated genes with the average coverage of the $S$. cerevisiae autosome. The read depth was calculated using the mpileup command from samtools. ${ }^{36}$

\section{ASSOCIATED CONTENT}

\section{S Supporting Information}

The Supporting Information is available free of charge on the ACS Publications website at DOI: 10.1021/acssynbio.7b00439. Supplementary figures and tables (PDF)

\section{AUTHOR INFORMATION}

\section{Corresponding Author}

*E-mail: mije@biosustain.dtu.dk.

\section{ORCID}

Tim Snoek: 0000-0002-5395-9034

Michael K. Jensen: 0000-0001-7574-4707

\section{Author Contributions}

TS, MKJ and JDK conceived this project. TS and DRS designed the experiments and carried out experimental work. FA performed whole-genome DNA sequencing and NGS data analysis. SS helped designing and carrying out repeated batch fermentations. MLS and JZ provided strains and materials. TS, DRS, MJK and JDK analyzed and interpreted the data. TS and $\mathrm{MKJ}$ wrote the paper, all authors assisted in this process.

\section{Notes}

The authors declare the following competing financial interest(s): JDK has a financial interest in Amyris, Lygos, Constructive Biology, and Demetrix.

\section{ACKNOWLEDGMENTS}

We are grateful to the Novo Nordisk Foundation for funding. In addition, we thank Alicia Viktoria Lis for consultancy regarding HPLC analysis and Christoffer Knudsen for assistance with the repeated batch fermentations.

\section{REFERENCES}

(1) Nielsen, J., and Keasling, J. D. (2016) Engineering Cellular Metabolism. Cell 164 (6), 1185-1197.

(2) Carlson, R. (2009) The Changing Economics of DNA Synthesis. Nat. Biotechnol. 27 (12), 1091-1094.

(3) Jakočiūnas, T., Jensen, M. K., and Keasling, J. D. (2015) CRISPR/Cas9 Advances Engineering of Microbial Cell Factories. Metab. Eng. 34, 44-59.

(4) Rogers, J. K., Taylor, N. D., and Church, G. M. (2016) BiosensorBased Engineering of Biosynthetic Pathways. Curr. Opin. Biotechnol. 42, 84-91.

(5) Fernandez-López, R, Ruiz, R., de la Cruz, F., and Moncalián, G. (2015) Transcription Factor-Based Biosensors Enlightened by the Analyte. Front. Microbiol. 6, 648.

(6) Lin, J.-L., Wagner, J. M., and Alper, H. S. (2017) Enabling Tools for High-Throughput Detection of Metabolites: Metabolic Engineering and Directed Evolution Applications. Biotechnol. Adv. 35 (8), 950970.
(7) Libis, V., Delépine, B., and Faulon, J.-L. (2016) Sensing New Chemicals with Bacterial Transcription Factors. Curr. Opin. Microbiol. 33, 105-112.

(8) Steensels, J., Snoek, T., Meersman, E., Picca Nicolino, M., Voordeckers, K., and Verstrepen, K. J. (2014) Improving Industrial Yeast Strains: Exploiting Natural and Artificial Diversity. FEMS Microbiol. Rev. 38 (5), 947-995.

(9) D'Ambrosio, V., and Jensen, M. K. (2017) Lighting up Yeast Cell Factories by Transcription Factor-Based Biosensors. FEMS Yeast Res., DOI: $10.1093 /$ femsyr/fox076.

(10) Wang, M., Li, S., and Zhao, H. (2016) Design and Engineering of Intracellular-Metabolite-Sensing/regulation Gene Circuits in Saccharomyces Cerevisiae. Biotechnol. Biotechnol. Bioeng. 113 (1), 206-215.

(11) Li, S., Si, T., Wang, M., and Zhao, H. (2015) Development of a Synthetic Malonyl-CoA Sensor in Saccharomyces Cerevisiae for Intracellular Metabolite Monitoring and Genetic Screening. ACS Synth. Biol. 4 (12), 1308-1315.

(12) Skjoedt, M. L., Snoek, T., Kildegaard, K. R., Arsovska, D., Eichenberger, M., Goedecke, T. J., Rajkumar, A. S., Zhang, J., Kristensen, M., Lehka, B. J., et al. (2016) Engineering Prokaryotic Transcriptional Activators as Metabolite Biosensors in Yeast. Nat. Chem. Biol. 12 (11), 951-958.

(13) Leavitt, J. M., Wagner, J. M., Tu, C. C., Tong, A., Liu, Y., and Alper, H. S. (2017) Biosensor-Enabled Directed Evolution to Improve Muconic Acid Production in Saccharomyces Cerevisiae. Biotechnol. J. 12, 1600687.

(14) Mahr, R., Gätgens, C., Gätgens, J., Polen, T., Kalinowski, J., and Frunzke, J. (2015) Biosensor-Driven Adaptive Laboratory Evolution of L-Valine Production in Corynebacterium Glutamicum. Metab. Eng. 32, 184-194.

(15) Esvelt, K. M., and Wang, H. H. (2013) Genome-Scale Engineering for Systems and Synthetic Biology. Mol. Syst. Biol. 9, 641.

(16) Dietrich, J. A., Shis, D. L., Alikhani, A., and Keasling, J. D. (2013) Transcription Factor-Based Screens and Synthetic Selections for Microbial Small-Molecule Biosynthesis. ACS Synth. Biol. 2 (1), 4758.

(17) Raman, S., Rogers, J. K., Taylor, N. D., and Church, G. M. (2014) Evolution-Guided Optimization of Biosynthetic Pathways. Proc. Natl. Acad. Sci. U. S. A. 111 (50), 201409523.

(18) Xiao, Y., Bowen, C. H., Liu, D., and Zhang, F. (2016) Exploiting Nongenetic Cell-to-Cell Variation for Enhanced Biosynthesis. Nat. Chem. Biol. 12 (5), 339-344.

(19) Umeyama, T., Okada, S., and Ito, T. (2013) Synthetic Gene Circuit-Mediated Monitoring of Endogenous Metabolites: Identification of GAL11 as a Novel Multicopy Enhancer of S-Adenosylmethionine Level in Yeast. ACS Synth. Biol. 2 (8), 425-430.

(20) Feng, J., Jester, B. W., Tinberg, C. E., Mandell, D. J., Antunes, M. S., Chari, R., Morey, K. J., Rios, X., Medford, J. I., Church, G. M., et al. (2015) A General Strategy to Construct Small Molecule Biosensors in Eukaryotes. eLife 4, 4.

(21) Kruyer, N. S., and Peralta-Yahya, P. (2017) Metabolic Engineering Strategies to Bio-Adipic Acid Production. Curr. Opin. Biotechnol. 45, 136-143.

(22) Niu, W., Draths, K. M., and Frost, J. W. (2002) Benzene-Free Synthesis of Adipic Acid. Biotechnol. Prog. 18 (2), 201-211.

(23) Weber, C., Brückner, C., Weinreb, S., Lehr, C., Essl, C., and Boles, E. (2012) Biosynthesis of Cis,cis-Muconic Acid and Its Aromatic Precursors, Catechol and Protocatechuic Acid, from Renewable Feedstocks by Saccharomyces Cerevisiae. Appl. Environ. Microbiol. 78 (23), 8421-8430.

(24) Curran, K. A., Leavitt, J. M., Karim, A. S., and Alper, H. S. (2013) Metabolic Engineering of Muconic Acid Production in Saccharomyces Cerevisiae. Metab. Eng. 15, 55-66.

(25) Suastegui, M., Matthiesen, J. E., Carraher, J. M., Hernandez, N., Rodriguez Quiroz, N., Okerlund, A., Cochran, E. W., Shao, Z., and Tessonnier, J.-P. (2016) Combining Metabolic Engineering and Electrocatalysis: Application to the Production of Polyamides from Sugar. Angew. Chem. 128 (7), 2414-2419. 
(26) Wach, A., Brachat, A., Pöhlmann, R., and Philippsen, P. (1994) New Heterologous Modules for Classical or PCR-Based Gene Disruptions in Saccharomyces Cerevisiae. Yeast 10 (13), 1793-1808.

(27) Cheng, T. H., Chang, C. R., Joy, P., Yablok, S., and Gartenberg, M. R. (2000) Controlling Gene Expression in Yeast by Inducible SiteSpecific Recombination. Nucleic Acids Res. 28 (24), $108 \mathrm{e}$.

(28) Piper, P., Calderon, C. O., Hatzixanthis, K., and Mollapour, M. (2001) Weak Acid Adaptation: The Stress Response That Confers Yeasts with Resistance to Organic Acid Food Preservatives. Microbiology 147 (Pt 10), 2635-2642.

(29) Maury, J., Germann, S. M., Baallal Jacobsen, S. A., Jensen, N. B., Kildegaard, K. R., Herrgård, M. J., Schneider, K., Koza, A., Forster, J., Nielsen, J., et al. (2016) EasyCloneMulti: A Set of Vectors for Simultaneous and Multiple Genomic Integrations in Saccharomyces Cerevisiae. PLoS One 11 (3), e0150394.

(30) Maddocks, S. E., and Oyston, P. C. F. (2008) Structure and Function of the LysR-Type Transcriptional Regulator (LTTR) Family Proteins. Microbiology 154 (Pt 12), 3609-3623.

(31) Jensen, N. B., Strucko, T., Kildegaard, K. R., David, F., Maury, J., Mortensen, U. H., Forster, J., Nielsen, J., and Borodina, I. (2014) EasyClone: Method for Iterative Chromosomal Integration of Multiple Genes in Saccharomyces Cerevisiae. FEMS Yeast Res. 14 (2), 238-248.

(32) Gietz, R. D., and Schiestl, R. H. (2007) Quick and Easy Yeast Transformation Using the LiAc/SS Carrier DNA/PEG Method. Nat. Nat. Protoc. 2 (1), 35-37.

(33) Jessop-Fabre, M. M., Jakočiūnas, T., Stovicek, V., Dai, Z., Jensen, M. K., Keasling, J. D., and Borodina, I. (2016) EasyClone-MarkerFree: A Vector Toolkit for Marker-Less Integration of Genes into Saccharomyces Cerevisiae via CRISPR-Cas9. Biotechnol. J. 11 (8), $1110-1117$

(34) Stovicek, V., Borodina, I., and Forster, J. (2015) CRISPR-Cas System Enables Fast and Simple Genome Editing of Industrial Saccharomyces Cerevisiae Strains. Metabolic Engineering Communications 2, 13-22.

(35) Jung, P. P., Christian, N., Kay, D. P., Skupin, A., and Linster, C. L. (2015) Protocols and Programs for High-Throughput Growth and Aging Phenotyping in Yeast. PLoS One 10 (3), e0119807.

(36) Li, H., Handsaker, B., Wysoker, A., Fennell, T., Ruan, J., Homer, N., Marth, G., Abecasis, G., and Durbin, R. (2009) 1000 Genome Project Data Processing Subgroup. The Sequence Alignment/Map Format and SAMtools. Bioinformatics 25 (16), 2078-2079. 